\title{
The Appearance of Congenitally Corrected Transposition of the Great Arteries on Myocardial Perfusion Imaging
}

\author{
Zaid Al-faham, Prashant Jolepalem, and Ching-Yee Oliver Wong \\ Department of Diagnostic Radiology and Molecular Imaging, Oakland University William Beaumont School of Medicine, Royal Oak, Michigan
}

\begin{abstract}
We present a case of incidentally discovered congenitally corrected transposition of the great arteries (ccTGA), initially seen on stress-rest myocardial perfusion imaging (MPI). ccTGA has a characteristic appearance on MPI, which reflects the functional alterations associated with this condition.
\end{abstract}

Key Words: cardiology (clinical); correlative imaging; MPI; transposition of the great arteries; ccTGA

J Nucl Med Technol 2015; 43:68-69

DOI: 10.2967/jnmt.114.139592

\section{W.}

e present a case of incidentally discovered congenitally corrected transposition of the great arteries (ccTGA), initially seen on stress-rest myocardial perfusion imaging (MPI).

\section{CASE REPORT}

A 30-y-old man with no known coronary history presented with chest pain at rest. The patient underwent test was normal without symptoms. The obtained SPECT images appeared to show a mirrored image of what would typically be expected (Fig. 1) in a normal heart (Fig. 2). This pattern suggested that there was preferential perfusion and increased mitochondrial uptake in the right ventricle likely secondary to the increased workload causing it to appear more prominent than the left ventricle. The only known cause of this appearance is ccTGA, which was confirmed by 2-dimensional echo. On the MPI, there was no evidence of stress-induced ischemia, and the right ventricular ejection fraction was normal.

\section{DISCUSSION}

ccTGA is a rare condition in which the atria are connected to their opposite ventricles because the ventricles are switched from their normal anatomic positions (Fig. 2). In this condition, the blood flows from the right atrium to the left ventricle on the right side, from which the pulmonary artery arises, and from the left atrium to the right ventricle

FIGURE 1. SPECT myocardial perfusion images demonstrate reversal of typical radiotracer distribution, with increased perfusion to right-sided ventricle, which is dilated to accommodate increased volume and thickened as result of increased afterload. Left-sided ventricle is smaller and has faint radiotracer uptake; therefore, its perfusion cannot be easily assessed.

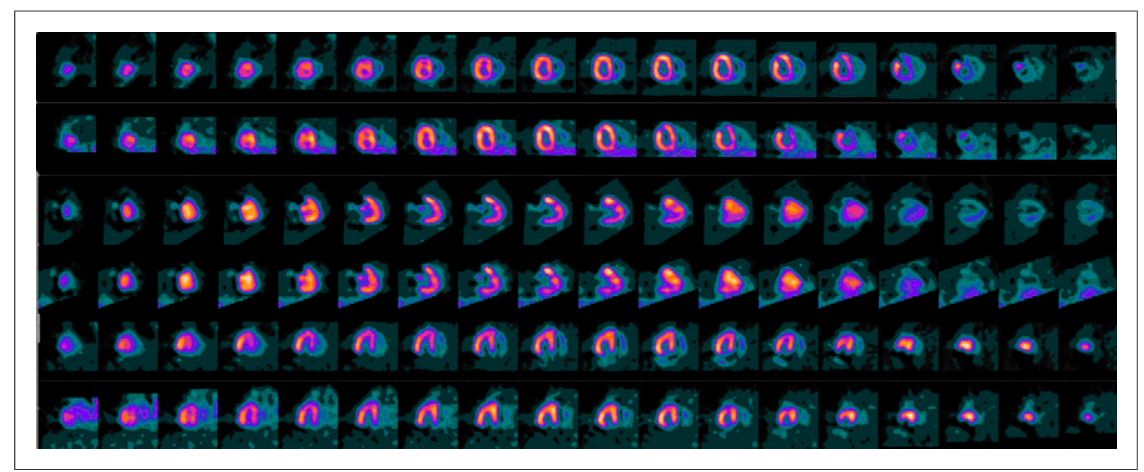

myocardial perfusion SPECT at rest and peak exercise using the 1-d protocol with 370 and $1,110 \mathrm{MBq}$ (10 and 30 $\mathrm{mCi}$ ) of ${ }^{99 \mathrm{~m}} \mathrm{Tc}-\mathrm{methoxy}$ isobutylisonitrile (sestamibi). The images were acquired using dedicated cardiac cameras with a $180^{\circ}$ arc in step-and-shoot mode. The cardiac stress

Received Feb. 24, 2014; revision accepted Apr. 21, 2014

For correspondence or reprints contact: Zaid Al-faham, Department of Diagnostic Radiology and Molecular Imaging, Oakland University William Beaumont School of Medicine, 3601 W. 13 Mile Rd., Royal Oak, MI 48073. E-mail: zaidfaham@gmail.com

Published online Aug. 28, 2014.

COPYRIGHT (c) 2015 by the Society of Nuclear Medicine and Molecular Imaging, Inc.

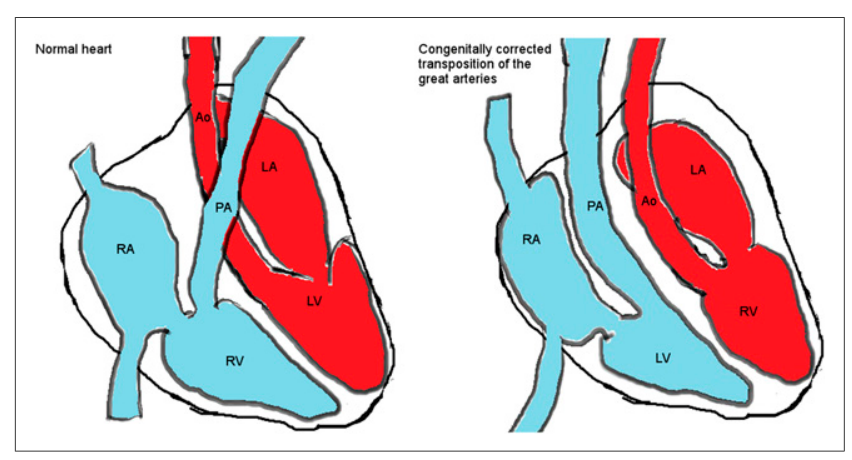

FIGURE 2. Anatomy and blood flow of ccTGA. 
on the left side, from which the aorta arises (1). Therefore, many patients develop right ventricular hypertrophy and failure because of the chronic exposure of the right ventricle to the high systemic blood pressure $(1,2)$. In patients with ccTGA, the frequency of right ventricular dysfunction after atrial switch varies from $8 \%$ to $16 \%$, and myocardial perfusion defects are common in long-term follow-up because the right ventricle was never intended to handle such high volumes or pressures (3).

ccTGA is associated with a reduced life expectancy due to ventricular septal defects $(74 \%)$, pulmonary valvular stenosis (74\%), systemic atrioventricular valve abnormalities (38\%), or complete heart block (5\%) (1). Lubiszewska et al. observed that myocardial perfusion defects are common in long-term follow-up after atrial switch. When ${ }^{99 \mathrm{~m}} \mathrm{Tc}-\mathrm{methoxyisobutyl-}$ isonitrile SPECT is used, the perfusion abnormalities were observed in $33 \%$ of the patients (3). The altered perfusion pattern seen on MPI is a result of the right ventricle assuming the usual function of the left ventricle.

Our default SPECT protocol uses the $180^{\circ} \mathrm{L}$-shaped dualdetector acquisition. However, it would have been better if we had performed the $360^{\circ}$ acquisition, especially in view of the relatively posterior location of right ventricle. The counts in the right ventricle in our case were much higher than those seen in the normal heart, and the information from our current SPECT scan may be adequate-except for possible minor distortion-compared with the complete $360^{\circ}$ acquisition.

\section{CONCLUSION}

ccTGA has a characteristic appearance on MPI in which the radiotracer distribution in the right ventricle is increased, with dilation and thickening of the right ventricle, and the left ventricle appears smaller than its normal size, with decreased radiotracer uptake.

\section{REFERENCES}

1. Caldarella C, Leccisotti L, Bruno I, Collarino A, Maggi F, Giordano A. Myocardial perfusion single-photon emission tomography (SPET) and positron emission tomography-computed tomography (PET-CT) imaging for congenitally corrected transposition of great arteries. Pediatr Cardiol. 2012;33:1435-1439.

2. Hornung TS, Bernard EJ, Jaeggi ET, Howman-Giles RB, Celermajer DS, Hawker RE. Myocardial perfusion defects and associated systemic ventricular dysfunction in congenitally corrected transposition of the great arteries. Heart. 1998;80:322-326

3. Lubiszewska B, Gosiewska E, Hoffman P, et al. Myocardial perfusion and function of the systemic right ventricle in patients after atrial switch procedure for complete transposition: long-term follow-up. J Am Coll Cardiol. 2000;36:13651370 . 